\title{
TRATAMIENTO DE LA LITIASIS VESICAL CON LÁSER
}

\author{
Miguel Arrabal Martín, Mercedes Nogueras Ocaña, Miguel Ángel Arrabal Polo, José Luís Miján Ortiz, \\ Francisco Valle Díaz de la Guardia y Armando Zuluaga Gómez.
}

Servicio de Urología. Hospital Universitario San Cecilio. Granada. España.

\begin{abstract}
Resumen.- OBJETIVO: El uso del láser para litotricia endoscópica, comienza en 1968 al utilizar Mulvaney un láser de rubí sin éxito, posteriormente se probaron láser de $\mathrm{CO} 2$ y Neodymio-YAG. Con el láser pulsado de colorante y el láser de alexandrita se obtienen unos rendimientos energéticos que oscilan de 30 a 200 ml, su capacidad de fragmentación no es universal y esta limitada a pequeños cálculos, generalmente ureterales, por lo que no han sido alternativa terapéutica en la litiasis vesical. El láser de Holmio genera pulsos de energía de 400-2500 ml, capaz de fragmentar todo tipo de cálculos. El Objetivo de este trabajo es analizar los resultados de la litotricia vesical endoscópica con láser de holmio YAG.
\end{abstract}

MÉTODOS: En el periodo de 2006-2008 hemos tratado 21 casos de litiasis vesical, con tamaños de $1 \mathrm{a} 4 \mathrm{~cm}$. en pacientes de 8-76 años, 6 mujeres y 15 hombres, que corresponden a: Cuatro casos de litiasis infantil, 3 de ácido úrico, 1 caso de cistina, litiasis de oxalato y/o fosfato cálcico en 7 casos, 5 casos de litiasis vesical sobre extremo inferior de doble jota, 1 caso de litiasis en ureterocele intravesical. El tratamiento se ha realizado con un equipo Dornier Medilas de 20 watios de Holmio-YAG como fuente de energía, que se ha aplicado a través de cistoscopios infantil-adulto o ureteroscopios de 7-8.5 Ch. semirrígido y flexible. El control post-operatorio se ha realizado con radiografía simple de aparato urinario y ecografía. Realizamos estudio de factores de riesgo litógeno y análisis de los fragmentos del cálculo.

RESULTADOS: Los 21 casos descritos corresponden a litiasis vesical secundaria o tipo II. En todos los casos se ha comprobado la ausencia de litiasis residual con estudios de imagen y se han corregido los factores de riesgo litógeno con procedimientos médicos o quirúrgicos.

CONCLUSIONES: Consideramos que hoy, la litotricia vesical endoscópica con láser de holmio si es alternativa terapéutica. A pesar de que existen múltiples opciones de tratamiento endoscópico, la litotricia transuretral con láser de holmio ofrece buenos resultados con un bajo porcentaje de complicaciones.

Palabras clave: Litiasis vesical. Litotricia endoscópica. Láser de Holmio. Láser.

Summary.- OBJECTIVES: The use of laser for endoscopic lithotripsy started in 1968 when Mulvaney tried a ruby laser without success; Later on, the CO2 laser and the Nd: YAG were tried. With the pulsed dye and alexandrite lasers energetic performances between 30 and $200 \mathrm{ml}$ are obtained, their capacity of fragmentation is not universal and is limited to small stones, generally ureteral stones, so that it has not been a therapeutic alternative for bladder lithiasis. The holmium laser 
generates energy pulses of $400-2500 \mathrm{~m}$, it is able to fragment every type of stone. The objective of this work is to analyze the results of endoscopic bladder lithotripsy with holmium-YAG laser.

METHODS: In the period between 2006-2008 we treated 21 cases of bladder lithiasis, with a stone size between 1 and $4 \mathrm{~cm}$ in patients from 8-76 years, six women and 15 men, which correspond to: four cases of infantile lithiasis, 3 of uric acid, one case of cystine, seven cases of calcium oxalate and/or phosphate, five cases of bladder lithiasis growing around a double J catheter, and one case of lithiasis within an intravesical ureterocele. Treatment was performed with a $20 \mathrm{~W}$ Dornier Medilas holmium-YAG equipment, applied using children/adult cystoscopes or 7-8.5 Ch ureteroscopes, both semirigid and flexible. Post operative control included KUB x-ray and ultrasound. We performed a study of lithogenic risk factors and stone fragments analysis.

RESULTS: The 21 cases described are all secondary or type II bladder lithiasis. In all cases the absence of residual lithiasis was checked with imaging studies and the lithogenic risk factors were corrected with medical or surgical procedures.

CONCLUSIONS: We consider that today bladder endoscopic lithotripsy with holmium laser is a therapeutic alternative. Despite there are multiple options for endoscopic treatment, transurethral lithotripsy with holmium laser offers good results with a low complication rate.

Keywords: Bladder lithiasis. Endoscopic lithotripsy. Holmium laser. Laser.

\section{INTRODUCCIÓN}

La litiasis urinaria ha cumplido un nuevo milenio, al menos 7000 años han transcurrido desde la formación de un cálculo vesical descubierto en una tumba prehistórica, por E. Smith en 1901 (1). Hasta el siglo XX, la litiasis vesical ha sido una enfermedad endémica en la vida humana, en el último siglo se ha producido un aumento de la incidencia de cálculos renales y ureterales en Europa y América, y disminución de los cálculos vesicales en relación con los progresos en dietética y nutrición, sin embargo en zonas con menor desarrollo social y económico persiste alta incidencia de litiasis vesical, especialmente en el sexo masculino, enfermos mayores de 50 años con patología obstructiva infravesical e infección urinaria (2).

Los cálculos vesicales suponen del 2 al $6 \%$ de la litiasis urinaria y en una proporción entre hombres y mujeres de $2 / 1$. La litiasis vesical endémica en el niño es más frecuente entre el primer y quinto año, para ir declinando progresivamente hasta los 10 años de vida, con claras diferencias geográficas y dietéticas (3).

\section{FACTORES FAVORECEDORES}

Las causas que se relacionan con la formación de los cálculos vesicales son, determinados hábitos de vida y alimentación, el estasis e infección urinaria, presencia de cuerpos extraños, desordenes metabólicos y alteraciones locales de la mucosa vesical. Las causas de la litiasis vesical infantil endémica se basan en la menor eliminación urinaria de fosfatos como consecuencia de una dieta escasa en proteínas animales, este déficit del sistema amortiguador de los fosfatos se compensa con una excesiva producción renal de $\mathrm{NH} 4$ que al unirse con ácido úrico forma urato amónico, además la alimentación vegetariana aumenta la eliminación urinaria de oxalato y la formación de oxalato cálcico (4).

El estasis urinario debido a obstáculos cervicoprostáticos y uretrales, y la disfunción vesical neurológica o por neovejiga intestinal acompañada o no de infección, son actualmente las causas más frecuentes de litiasis vesical (5). Los cuerpos extraños vesicales, secundarios a procedimientos diagnósticos o terapéuticos sobre el aparato urinario, emigrados desde otros órganos o introducidos por la uretra por aberraciones o equivocaciones sexuales, pueden constituir el núcleo del cálculo (6-7).

El hiperparatiroidismo, hiperuricosuria, cistinuria, etc., asociados a retención urinaria, pueden dar lugar a una precipitación cristalina y formación de un cálculo vesical, en la serie de Bergman el $20 \%$ de los cálculos vesicales se relacionaron con una o varias alteraciones metabólicas (8). Otros factores que pueden tener influencia en la génesis de los cálculos o favorecer su formación, están en relación con alteraciones locales de la mucosa vesical, por traumatismos, neoformaciones, enfermedades parasitarias, lesiones radicas, etc. (9).

\section{CLASIFICACIÓN}

Desde el punto de vista etiopatogénico, los cálculos vesicales se clasifican en primarios o migratorios y secundarios o primitivos (4).

a/ Cálculos primarios o migratorios. Se consideran cálculos primarios, a los de origen renal, descendidos a lo largo del conducto ureteral y detenidos en la cavidad vesical, donde pueden seguir creciendo lentamente.

b/ Cálculos secundarios o primitivos. Se consideran cálculos secundarios a los que tienen un origen primitivo en la cavidad vesical, secundario a obstrucción del tracto urinario inferior, infección urinaria, cuerpos extraños, disfunciones vesicales, etc. También se incluyen en este grupo a los cálculos originados por la asociación de una alteración metabólica y residuo urinario postmiccional, considerados por algunos autores como cálculos metabólicos.

Desde el punto de vista clínico-terapéutico los cálculos vesicales se clasifican según su tamaño y consistencia en tipo I y II (Tabla I). Pertenecen al tipo I, todos los cálculos vesicales primarios con diámetro menor de $2-3 \mathrm{~cm}$. $(2,6-3$ $\mathrm{cm}^{2}$ ) dependiendo de su consistencia (Mayor consistencia 
TABLA I. RELACIÓN TAMAÑO Y SUPERFICIE DE LOS CÁLCULOS URINARIOS.

\begin{tabular}{|l|l|}
\hline TAMAÑO & SUPERFICIE \\
\hline $0-10 \mathrm{~mm}$ & $0-100 \mathrm{~mm}^{2}$ \\
\hline $10-20 \mathrm{~mm}$ & $100-260 \mathrm{~mm}^{2}$ \\
\hline $20-30 \mathrm{~mm}$ & $260-300 \mathrm{~mm}^{2}$ \\
\hline $30-40 \mathrm{~mm}$ & $300-700 \mathrm{~mm}^{2}$ \\
\hline$>40 \mathrm{~mm}$ & $>700 \mathrm{~mm}^{2}$ \\
\hline
\end{tabular}

/ menor tamaño) (Tabla II). Se incluyen en el tipo II, en general todos los cálculos vesicales secundarios y los primarios mayores de $2.6-3 \mathrm{~cm}^{2}(10)$.

\section{RECOMENDACIONES TERAPÉUTICAS}

El tratamiento de la litiasis vesical es tan antiguo como el conocimiento de la propia enfermedad, que se remonta al antiguo Egipto. La litotomía perineal practicada por Susruta, 500 años a. de C., y descrita exhaustivamente por Celso en el primer siglo de nuestra era, se considera como la más antigua técnica quirúrgica y ha sido utilizada hasta el año 1700 (1). En el siglo XVIII, Pierre Franco describe la talla lateral y posteriormente la talla suprapúbica, técnica que se impone definitivamente a partir del siglo XIX. En este siglo, con las aportaciones de Civiale y Max
Nitze nace la litotricia transuretral y comienza el fin de los XXIV siglos de talla vesical (11). Los primeros ensayos de tratamiento médico para disolver los cálculos vesicales, fueron realizados por Avicena en el siglo XI, instilando en la vejiga diversas sustancias a través de un catéter (12). En la primera mitad del siglo XX, con la litotricia vesical endoscópica, renace el interés por la litolisis local, irrigando los cálculos vesicales con diversas soluciones (Renacidin, solución Suby $G \circ M$, Alcalinos) con rentabilidad clínica limitada (13), no obstante en pacientes con sonda permanente o autocateterismo intermitente puede ser útil la irrigación preventiva dos o tres veces al día (Suero salino, Renacidin, ácido acético al 0.25-0.5\%). Actualmente el tratamiento de la litiasis vesical debe contemplar no sólo la exéresis del cálculo, sino también la corrección de los factores etiopatogénicos.

\section{Litotricia Extracorpórea}

El método de la litotricia extracorpórea con ondas de choque comienza a aplicarse en la década de 1980, en cálculos renales menores de $2 \mathrm{~cm}$., sus indicaciones se extienden de forma progresiva a cálculos ureterales, cálculos más complejos e incluso a cálculos vesicales, en esta década en España se tratan 72 casos de litiasis vesical, recogidos en un estudio multicéntrico sobre 25.000 pacientes de litiasis urinaria tratados en 17 centros sanitarios. Los resultados iniciales recomiendan la aplicación del método en cálculos vesicales tipo I (4-14). En el estudio multicéntrico realizado al final del siglo XX sobre 300.000 casos 163 centros españoles) el $1 \%$ corresponden a litiasis vesicales, no se ratifica la prioridad de la litrotricia vesical extracorpórea sobre la litotricia endoscópica (15) y las referencias bibliográficas no superan la decena. Bathia y Biyani consideran que la litotricia extracorpórea es una modalidad simple, eficaz y segura en el tratamiento de la litiasis vesical sin necesidad de anestesia y menor morbilidad que la litotricia endoscópica, con buenos resultados en cálculos

TABLA II. RELACIÓN CONSISTENCIA Y COMPOSICIÓN DE LOS CÁLCULOS URINARIOS.

\begin{tabular}{|c|l|}
\hline CONSISTENCIA & \multicolumn{1}{|c|}{ COMPOSICIÓN DEL CÁLCULO } \\
\hline DUROS & $\begin{array}{l}\text { Ácido úrico, Cistina, Brushita, Oxalato cálcico monohidratado de gran masa litiásica } \\
\text { - radiodensidad alta. }\end{array}$ \\
\hline INTERMEDIOS & $\begin{array}{l}\text { Oxalato cálcico monohidratado de radiodensidad media, Fosfato amónico magnésico } \\
\text { de gran masa radiodensa. }\end{array}$ \\
\hline BLANDOS & $\begin{array}{l}\text { Fosfatos cálcicos de radiodensidad media, Oxalato cálcico dihidratado de radiodensi- } \\
\text { dad porosa, Fosfato amónico magnésico (FAM) radiodensidad baja. }\end{array}$ \\
\hline MUY BLANDOS & $\begin{array}{l}\text { FAM puro con mucoproteína. No posee estructura cristalina. (LEOC no es efectiva al } \\
\text { no existir prácticamente interfase entre cálculo y tejidos) }\end{array}$ \\
\hline
\end{tabular}


menores de $25 \mathrm{~mm}(16)$, Kojima y cols. la incluyen como buena opción terapéutica, aunque pueden ser necesarios múltiples tratamientos y maniobras auxiliares para dejar al paciente libre de cálculos (17), Delakas y cols. tratan 52 pacientes con cálculos de 10-22 mm de diámetro, sin anestesia ni hospitalización y refieren un $94 \%$ de buenos resultados, recomiendan el método especialmente en pacientes de alto riesgo quirúrgico (18), en una serie de 183 casos Millan Rodríguez y cols., obtienen resultados similares en cálculos menores de $2 \mathrm{~cm}^{2}$, en cálculos de $2-3 \mathrm{~cm}^{2}$ el éxito desciende al $57 \%$ y en cálculos $>3 \mathrm{~cm}^{2}$ al $42 \%$. En niños generalmente el procedimiento no se puede realizar sin anestesia y Duarte y cols. refieren un éxito global (20) del $60 \%$, y debemos valorar la posibilidad de secuelas en el aparato genital, según los hallazgos de estudios experimentales (21).

\section{Cirugía Endoscópica}

La cirugía endoscópica permite la exéresis del cálculo y la corrección simultánea de los factores obstructivos. En el siglo XIX Civiale realiza la primera litotricia funcional con instrumentos mecánicos y Bigelow introduce la litopalaxia mediante un litotritor ciego que consigue la fragmentación del cálculo y evacuación de los fragmentos (11), con los litotritores ópticos de Reuter, Maver-Mayer, Storz, se mejoran los resultados y descienden las complicaciones. En 1955 se desarrolla la litotricia electrohidráuli$\mathrm{ca}$, que fue considerada como un gran avance tecnológico de alta efectividad (22), lo que permite tratar cálculos de gran masa litiásica (23) hasta $3-4 \mathrm{~cm}$., con algunos efectos adversos, como lesiones de la mucosa, por lo que se contraindica en pacientes con infección urinaria, microvejiga y litiasis infantil, en cálculos duros la fragmentación es incompleta. En 1970 se introduce la litotricia ultrasónica, basada en el fenómeno físico de la piezoelectricidad, que tiene como ventaja sobre la litotricia electrohidraúlica el que no lesiona la mucosa vesical y aspira simultáneamente los fragmentos litiásicos, de gran utilidad en cálculos grandes de consistencia moderada, algunos autores la utilizan a través de un trocar suprapúbico en el tratamiento de la litiasis infantil (24), como alternativa a la cirugía abierta. La litotricia a través del trocar suprapúbico es más rápida y eficaz que la litotricia transuretral, por lo que se recomienda en el tratamiento combinado de adenoma de próstata y gran litiasis vesical, > de 3-4 cm. (5-25).

En la década de 1990 se desarrolla la energía cinética, a partir de aire a presión de la red hospitalaria conectado al equipo se generan ondas de energía que se transmiten al cálculo a través de una sonda metálica semirrigida of flexible, el sistema fue desarrollado por Electro $\mathrm{Me}$ dical System en la Clínica Universitaria de Lausana (26). El Lithoclast suizo tiene alta capacidad de fragmentación y se considera un método simple y seguro en el tratamiento de todo tipo de cálculos vesicales (27), su mayor inconveniente radica en la posibilidad de desplazamiento de la litiasis al contacto con la sonda, la asociación de un sistema de aspiración (Lithovac) para su uso a través del cistoscopio mejora el proceso y consigue a través de sus sondas de 10-12 Ch. la aspiración de fragmentos litiásicos de hasta $3.5 \mathrm{~mm}$. La litotricia electrocinética (litotritor EKL-compact) se basa en el mismo principio de transmisión de la energía cinética del sistema anterior neumocinético, pero en lu- gar de aire comprimido utiliza electroimanes para generar las ondas de presión, su utilización es todavía más simple e igualmente segura y eficaz, especialmente en cálculos grandes y duros (28). Recientemente se ha desarrollado un nuevo litrotritor neumocinético, más simple, compacto y ergonómico, el StoneBreaker genera ondas de alta presión que producen una fragmentación rápida por contacto y mínimo desplazamiento del cálculo, sin lesiones del urotelio (29), con una efectividad en la fragmentación de los cálculos vesicales del $100 \%$.

El uso del láser para litotricia endoscópica, comienza en 1968 al utilizar Mulvaney un láser de rubí en un estudio experimental sin éxito, posteriormente se probaron láseres de $\mathrm{CO}_{2}$ y Neodymio-YAG (28). El láser pulsado de colorante (cumarina), desarrollado por la Corporación Candela en USA, consiguió generar una energía suficiente para el tratamiento endoscópico de la litiasis (30), posteriormente se han desarrollado láseres de Alexandrita y de Holmio-YAG. Con el láser pulsado de colorante y el láser de alexandrita se obtienen unos rendimientos energéticos que oscilan de 30 a $200 \mathrm{~mJ}$, su capacidad de fragmentación no es universal y esta limitada a pequeños cálculos, generalmente ureterales, por lo que no han sido alternativa terapéutica en la litiasis vesical (31). El láser de Holmio emite un haz de fotones con longitud de onda de 2090 $\mathrm{nm}$, que genera pulsos de 350 microsegundos y energía de 400-2500 mJ, capaz de fragmentar todo tipo de cálculos con independencia de composición, color, tamaño, y localización, puede usarse con instrumentos rígidos y flexibles. El Objetivo de este trabajo es analizar los resultados de la litotricia vesical endoscópica con láser de holmio YAG.

\section{MATERIAL Y MÉTODOS}

En el periodo de 2006-2008 hemos tratado 21 casos de litiasis vesical, con tamaños de 1 a $4 \mathrm{~cm}$. en pacientes de 8-76 años, 6 mujeres y 15 hombres, que corresponden $a$ :

Cuatro casos de litiasis vesical infantil en pacientes de 8-15 años, tres pacientes con litiasis de ácido úrico, un caso de litiasis ureteral y vesical de cistina, litiasis de oxalato y/o fosfato cálcico en 7 casos, cinco casos de litiasis vesical sobre extremo inferior de doble jota, un caso de litiasis en ureterocele intravesical. El diagnostico se ha realizado con radiografía simple, ecografía en 17 casos y urograma en 4 casos. El tamaño de los cálculos se ha determinado con las pruebas de imagen y la composición por sus características radiológicas y ecográficas, datos clínicos y bioquímicos.

El tratamiento se ha realizado con un equipo Dornier Medilas de 20 watios de Holmio-YAG como fuente de energía, que se ha aplicado a través de cistoscopios infantil-adulto o ureteroscopios de 7-8.5 Ch. semirrigido y flexible. En el equipo Dornier Medilas de 20 watios, el elemento emisor del láser es un cristal de Ytrio-AluminioGranate dopado con Holmio, su activación produce un haz de fotones infrarrojo que se transmite por fibras de cuarzo de 270-400-600 micras a una longitud de onda de $2090 \mathrm{~nm}$ (2.1 micrones), su cromóforo es el agua por lo que se ha aplicado por contacto para evitar su absorción 
por el líquido de irrigación, genera una energía de 400$2500 \mathrm{~mJ}$ por pulsos de 350 microsegundos con frecuencia de 3-12 hertzios, su efecto fototérmico y fotomecánico por expansión térmica vaporiza y fragmenta el cálculo, aunque su penetración tisular es de $0.3 \mathrm{~mm}$ hemos evitado su aplicación sobre el urotelio para evitar efectos colaterales. El tratamiento se ha realizado bajo analgesia-sedación ambulatoria en adultos y anestesia general y hospitalización en dos niños.

El control post-operatorio se ha realizado con radiografía simple de aparato urinario y ecografía. Realizamos estudio de factores de riesgo litógeno y análisis de los fragmentos del cálculo. Además se ha realizado el tratamiento correspondiente de los factores litogénicos.

\section{RESULTADOS}

Los 4 casos de litiasis infantil corresponden a niña de 12 años y niño de 8 años con cálculos de oxalato cálcico y urato amónico secundarios a dieta vegetariana baja en proteínas (Figuras 1 - 3), enferma de 15 años con litiasis de fosfato cálcico antecedentes de mielomeningocele y enterocistoplastia en programa de autocateterismo (acidosis metabólica, hipercalciuria y $\mathrm{pH}$ urinario alcalino) (Figura 4), paciente de 12 años con litiasis de fosfato cálcico ante- cedentes de hipospadias y diagnosticado de acidosis tubular renal distal. En las tres niñas se ha realizado lasertricia con holmio a través de un ureteroscopio del $8.5 \mathrm{Ch}$, en el paciente de 12 años se ha utilizado un ureteroscopio del 7 Ch y sonda suprapúbica para mantener el flujo del líquido de irrigación. Los dos cálculos de oxalato y urato amónico se fragmentan con facilidad, se ha realizado litotricia laminar con pulverización y lavado de cavidad vesical hasta comprobar la ausencia de residuos, en los cálculos de fosfato se produce mayor vaporización y pulverización que fragmentación, la fibra tiende a perforar el cálculo, los pequeños fragmentos se han retirado con pinzas.

Los 3 casos de litiasis de ácido úrico corresponden a tres hombres que presentan hiperplasia prostática, los cálculos se han fragmentado sin dificultad, mínima pulverización (Figura 5). Hemos asociado quimiolisis oral con citrato potásico y dieta baja en purinas, en dos casos se ha realizado resección endoscópica de la próstata y en un caso tratamiento médico con tamsulosina.

El caso de litiasis de cistina corresponde a un hombre de 34 años, familia de Harris tipo I (hermana con litiasis renoureteral bilateral, cirugía percutánea, ureteroscopia y LEOC), se ha realizado lasertricia vesical y ureteral y profilaxis con dieta hídrica hiposódica y alcalinizantes urinarios.

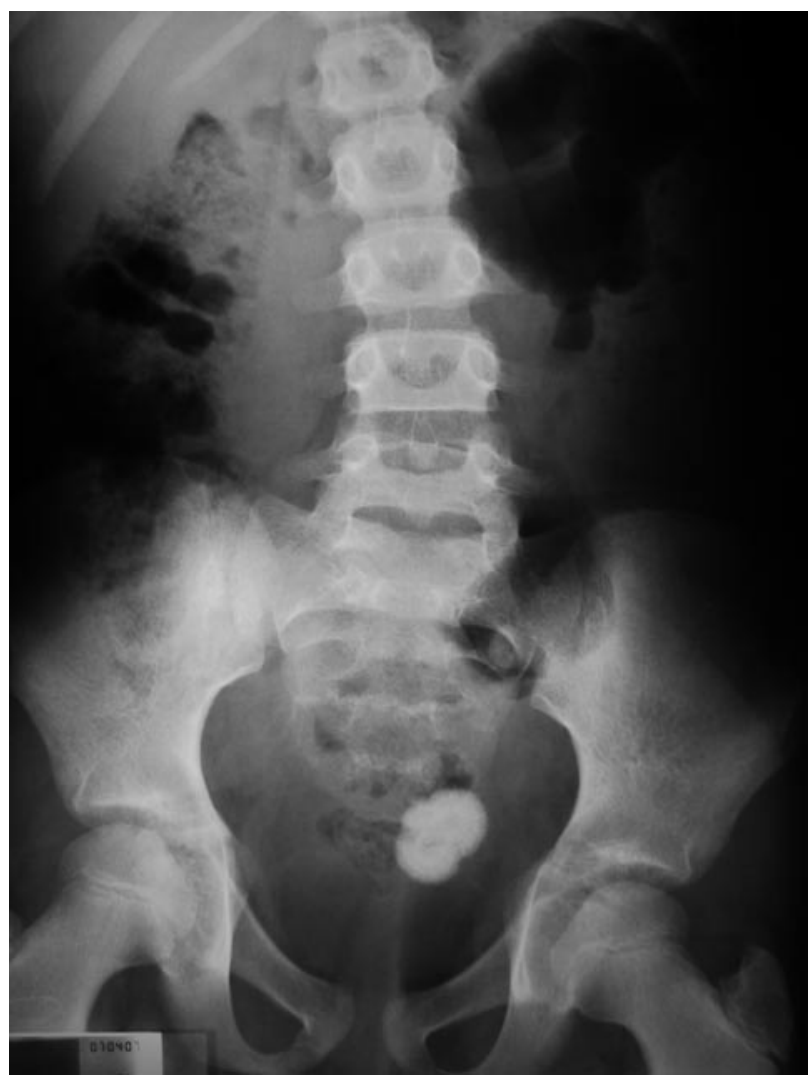

FIGURA 1. Litiasis vesical infantil de oxalato cálcico y urato amónico.

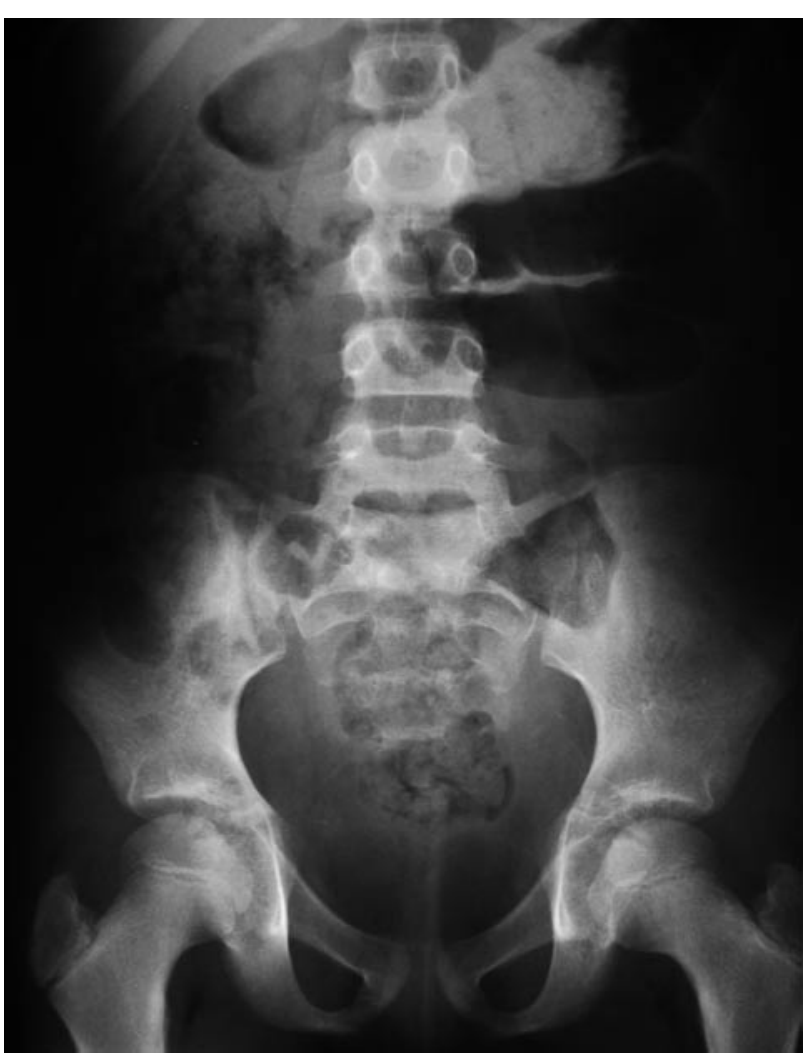

FIGURA 2. Rx simple a las 24 horas de lasertricia con holmio sobre el cálculo de la figura 1 . 


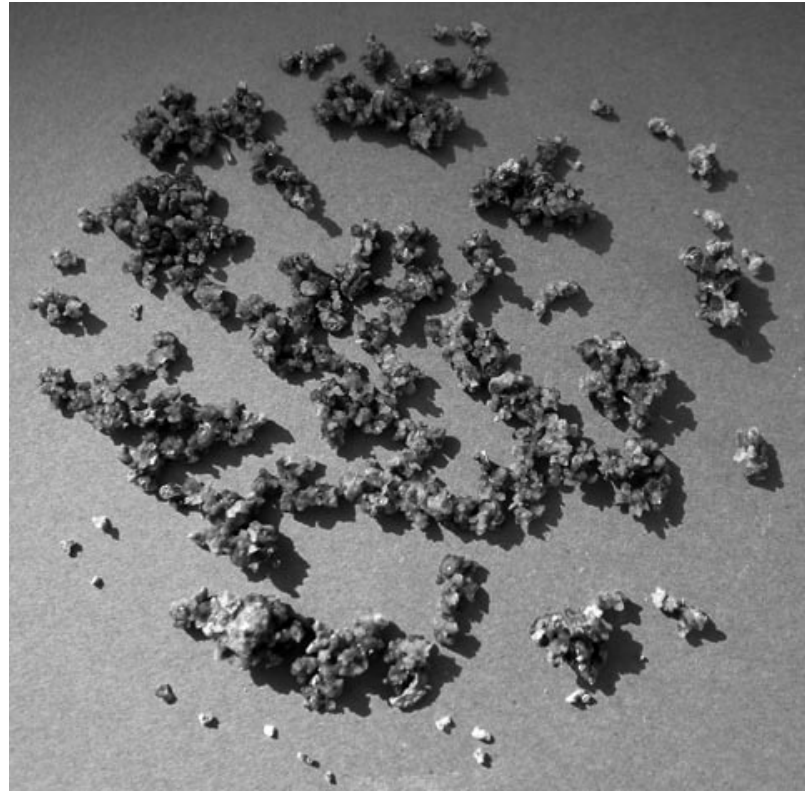

FIGURA 3. Fragmentos del cálculo de la figura 1, tras pulverización con láser de holmio.

Los 7 casos de litiasis cálcica corresponden a seis hombres con obstrucción del tracto urinario inferior $(5 \mathrm{ca}$ sos de HBP y 1 caso de estenosis de uretra), un paciente con hiperparatiroidismo. Se trata de tres casos con cálculos de oxalato cálcico, dos de oxalato y ácido úrico y dos de oxalato y fosfato cálcico (Figura 6). El oxalato cálcico monohidratado se fragmenta con mínima pulverización (2500 $\mathrm{mJ}$ a $3-5 \mathrm{~Hz}$ ), el oxalato cálcico dihidratado se fragmenta sin dificultad pero con mayor pulverización por lo que se necesita aumentar el flujo del líquido de irrigación, la mayor pulverización se produce con el fosfato cálcico, es necesario realizar lavado con suero salino para facilitar

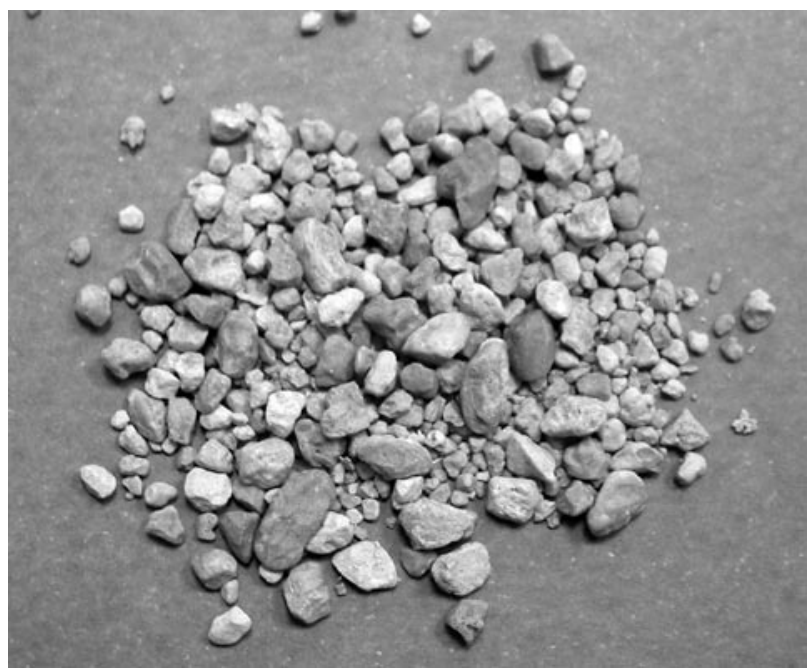

FIGURA 5. Fragmentos de cálculo de ácido úrico tras fragmentación con láser.

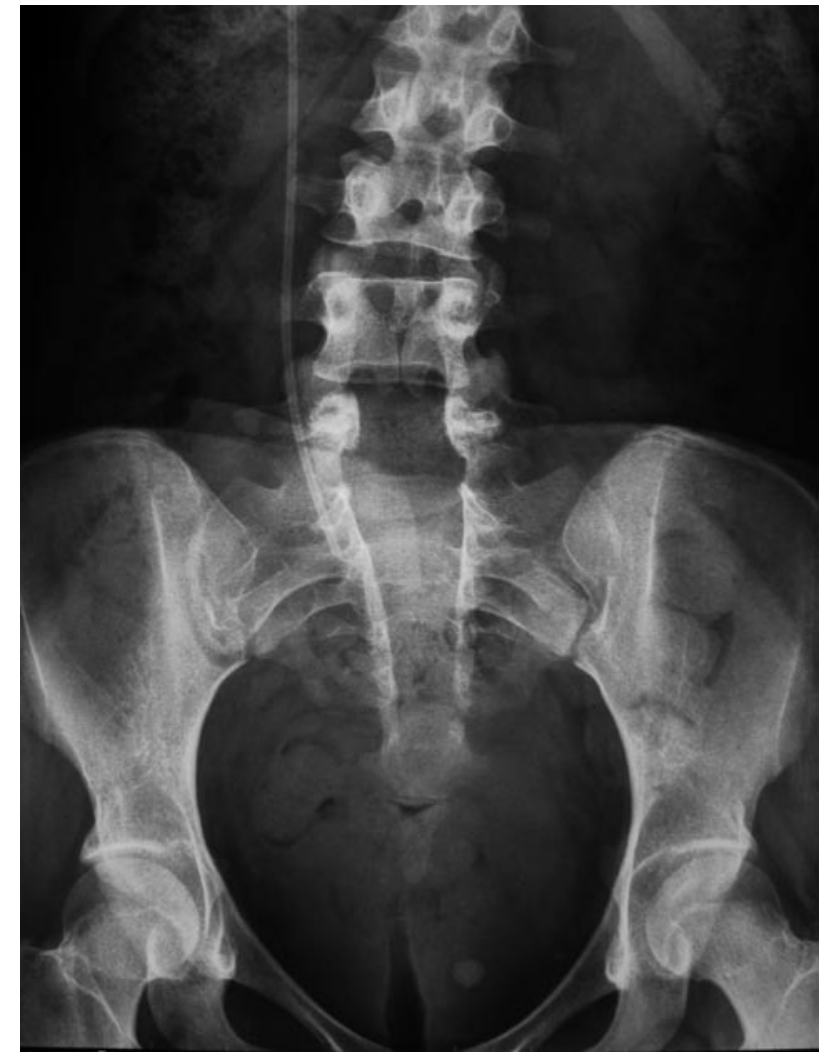

FIGURA 4. Litiasis de fosfato cálcico en enterocistoplastia.

la visón endoscópica. En tres casos se ha realizado resección endoscópica de la próstata, en un caso uretrotomía endoscópica, en dos casos tratamiento con inhibidores de la 5-alfa reductasa y en un caso paratiroidectomía.

Los 5 casos de litiasis por calcificación del extremo inferior del catéter doble jota, corresponden a tres mujeres en tratamiento con ondas de choque por litiasis renal de cistina, cálcica con hipercalciuria renal y de struvita; y dos hombres en tratamiento con ondas de choque por litiasis renal cálcica que se ha complicado con infección urinaria (Figura 7).

El último caso corresponde a una mujer de 54 años diagnosticada de ureterocele intravesical con litiasis gigante de oxalato cálcico de $2.5 \times 4 \mathrm{~cm}$., se ha realizado meatotomía oblicua con láser de holmio $(2000 \mathrm{~mJ}$ a $10 \mathrm{~Hz}$ ) y lasertricia a través de ureteroscopio de $8.5 \mathrm{Ch}$. fragmentación con mínima vaporización y pulverización, extracción de fragmentos con pinzas, en el estudio urográfico de control no se observa litiasis residual y excelente morfología de la vía excretora (Figuras 8 y 9).

Los 21 casos descritos corresponden a litiasis vesical secundaria o tipo II. En todos los casos se ha comprobado la ausencia de litiasis residual con estudios de imagen y se han corregido los factores de riesgo litógeno con procedimientos médicos o quirúrgicos. 


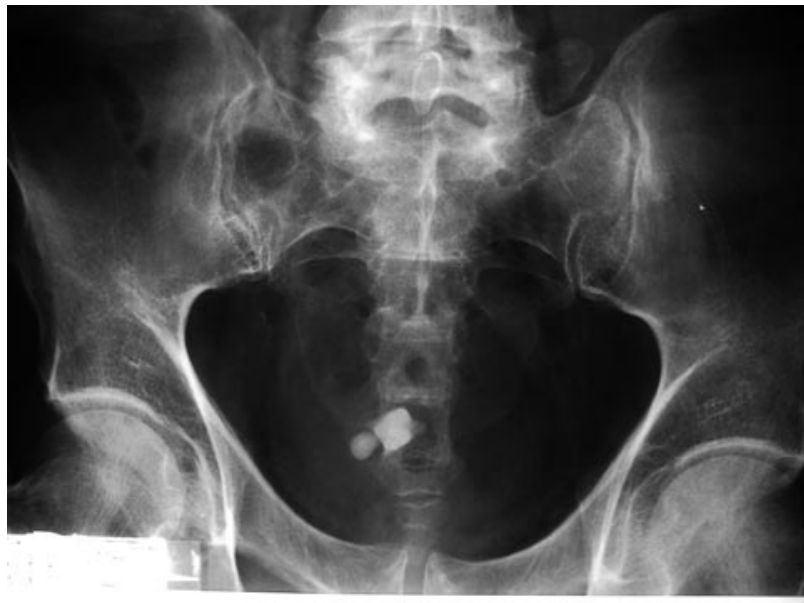

FIGURA 6. Litiasis vesical de oxalato y fosfato cálcico.

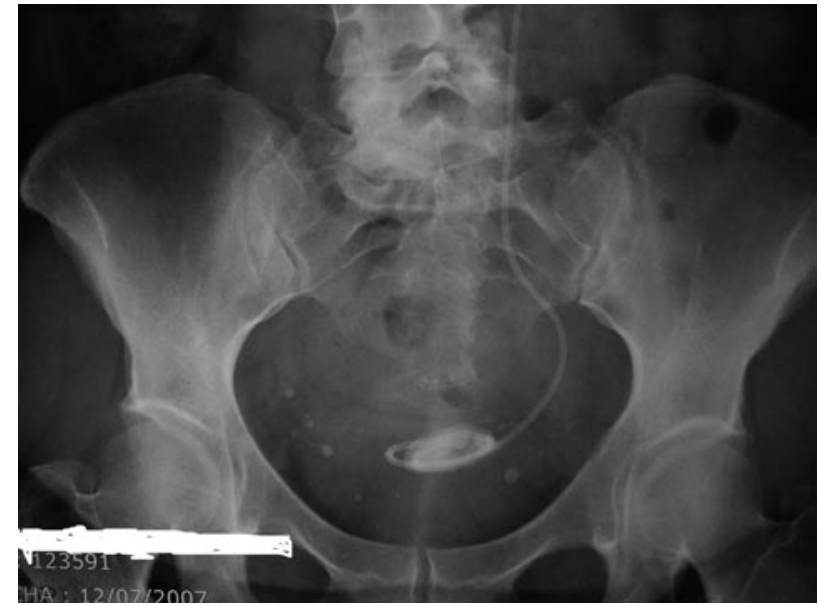

FIGURA 7. Litiasis de estruvita sobre extremo vesical de catéter ureteral con doble jota.

\section{DISCUSIÓN}

Tras las investigaciones iniciales con láser de Rubí, $\mathrm{CO}_{2}$, Neodymio-YAG, se consigue implantar la lasertricia in vivo con láser pulsado de colorante-cumarina, haz de luz verde con longitud de onda entre $504-514 \mathrm{~nm}$ que genera una energía de $60-200 \mathrm{~mJ}$ en pulsos de 1-2 microsegundos, se aplica al cálculo con fibras de cuarzo de 200-600 micras recubiertas de silicona a una frecuencia de $5-20 \mathrm{~Hz}$, la absorción de la energía provoca ondas acústicas que fragmentan el cálculo con mínimos efectos sobre el urotelio (32). Algunos cálculos absorben muy poco esta longitud de onda, como los de cistina, y la fragmentación es poco efectiva y poco rentable en cálculos grandes. Con el objetivo de mejorar estos resultados, en la década de 1990 se comienza a utilizar el láser de Alexandríta, láser sólido con longitud de onda de $750 \mathrm{~nm}$, que genera pulsos de energía de 30-120 mJ, el cromóforo de este láser es la melanina, por lo que su absorción en los cálculos es irregular (33-34), se fragmentan con dificultad los cálculos de fosfato y ácido úrico (31). La rentabilidad de estas fuentes de energía en la fragmentación de los cálculos vesicales ha sido baja y no competitiva con otras alternativas de litotricia vesical endoscópica, electrohidráulica, ultrasóni$c a$, cinética. Actualmente con la energía neumocinética del equipo stonebreaker (29), se consigue la fragmentación de cualquier tipo de litiasis con independencia de su composición y tamaño, se trata de una energía cinética de alta presión, 30 bares en el cálculo, que se aplica por contacto con una sonda metálica semirrigida, no lesiona el urotelio y a diferencia de otros sistemas de energía cinética produce mínima retropulsión del cálculo, aún no se dispone de sondas flexibles.

El láser de Holmio emite un haz de fotones con longitud de onda de $2090 \mathrm{~nm}$, que genera pulsos de 350 microsegundos y energía de 400-2500 mJ, capaz de fragmentar todo tipo de cálculos con independencia de composición, color, tamaño, y localización, puede usarse con instrumentos rígidos y flexibles con fibras de 270-600 micras. El haz de holmio es fuertemente absorbido por el agua, por

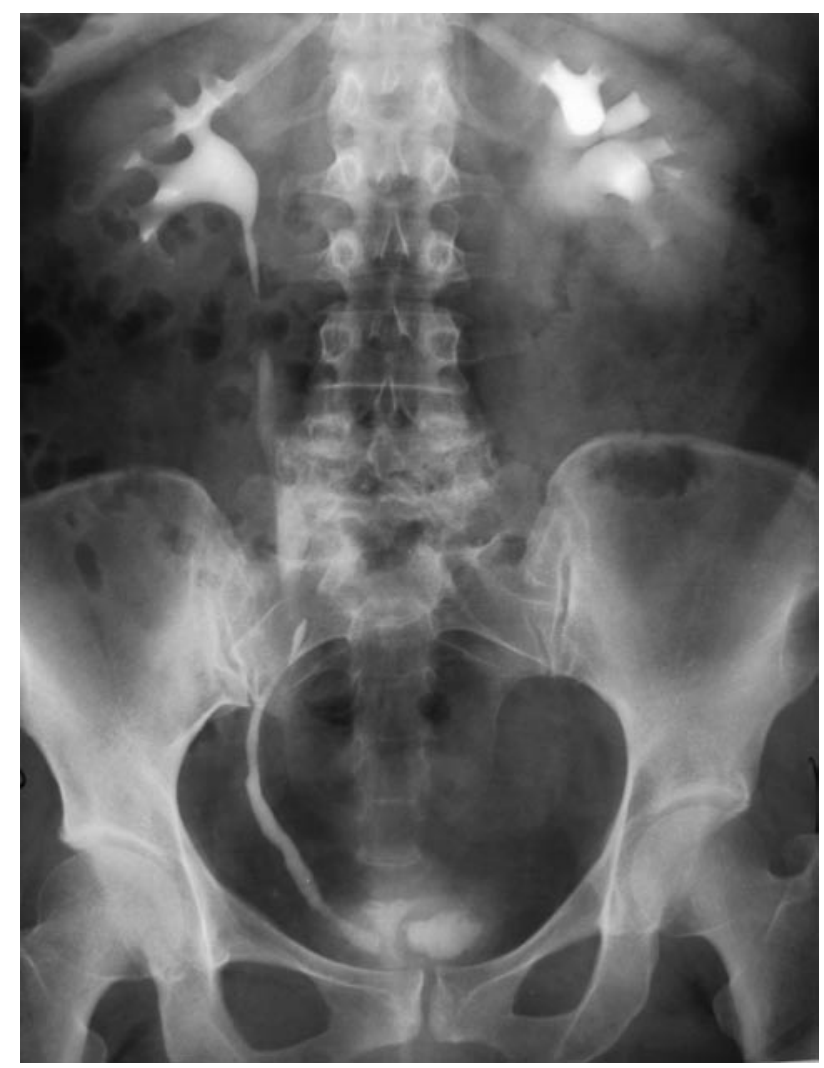

FIGURA 8. Litiasis de oxalato cálcico en ureterocele

FIGURA 8. Litiasis de oxalato cálcico en ureterocele
intravesical.

tanto se aplica por contacto de la fibra sobre el cálculo, la absorción de la energía produce una expansión térmica que fragmenta y vaporiza el cálculo con mayor efectividad y menor retropulsión y complicaciones que otros sistemas de láser como el de Alexandrita (31) o el de Neodimio- 


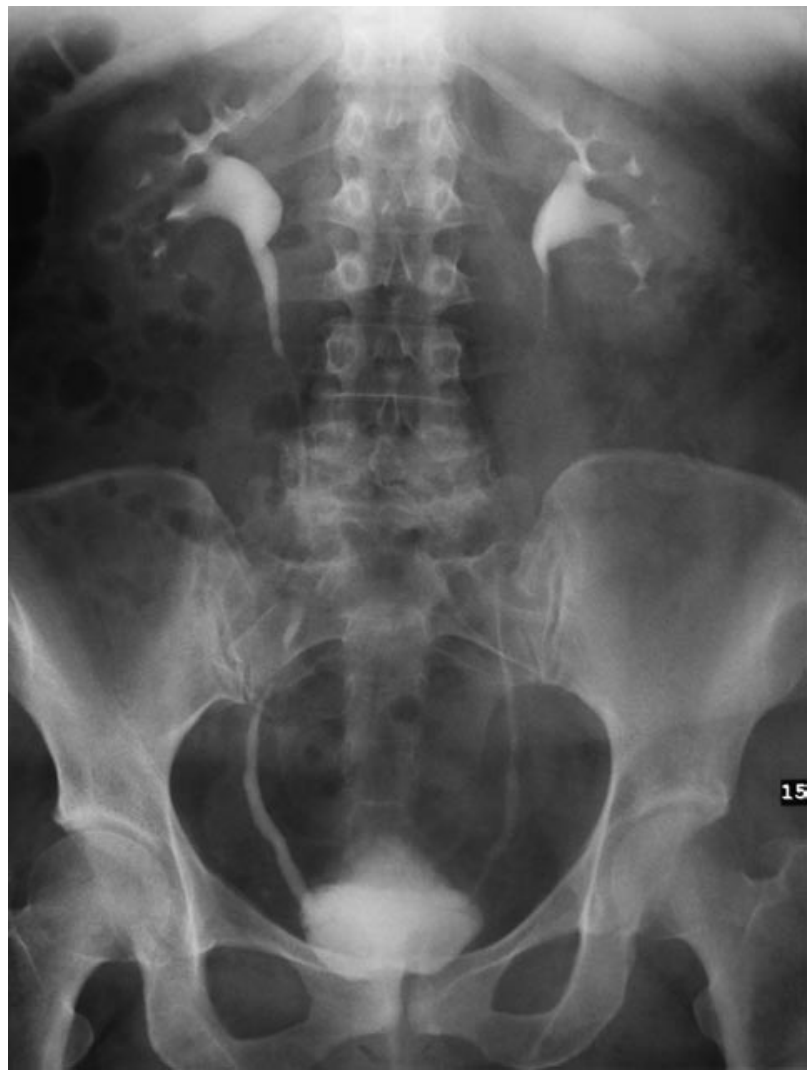

FIGURA 9. Estudio de control urográfico tras meatotomía oblicua del ureterocele y litotricia con holmio.

YAG con doble pulso (láser FREDDY) (35). Con el láser de Holmio se puede realizar tratamiento simultáneo de otras patologías relacionadas con la litiasis vesical, como la obstrucción del tracto urinario inferior por adenoma de próstata, esclerosis de cuello vesical o estenosis de uretra (36), Shah y cols. han tratado a 32 pacientes diagnosticados de hiperplasia prostática (1 1-172 gramos, media de 51.9 g) y litiasis vesical de $34.6 \mathrm{~mm}$ (rango de $12.70 \mathrm{~mm}$ ) de diámetro, realizan enucleación prostática (HoLEP) y litotricia transuretral con láser de holmio, todos los pacientes quedan libres de litiasis y el peso del tejido prostático enucleado es de $34.6 \mathrm{~g}(5-88 \mathrm{~g})$ con tiempos de intervención de 40230 minutos (media de 97.7 minutos) y complicaciones intraoperatorias y postoperatorias en $12.5 \%$ y $15.6 \%$ de los pacientes, consideran la morbilidad aceptable, Aron y cols en cálculos $>3 \mathrm{~cm}$. recomiendan la cistolitotricia cinética por trocar suprapúbico simultánea a la resección prostática, para disminuir el tiempo de intervención y bajar la morbilidad (25). Considerando que la litotricia vesical se puede realizar bajo analgesia y sedación como procedimiento ambulatorio, en los casos de gran masa litiásica se puede bajar la morbilidad si ambos procedimientos se realizan de forma independiente (5). En la cistolitotricia suprapúbica generalmente se utiliza la misma técnica que en la nefrolitotomía percutánea con extracción de grandes fragmentos a través de la vaina de Amplatz, el doble abordaje transuretral y suprapúbico permite trabajar con mayor flujo y mejor visión, utilizar instrumental de mayor calibre y efecti- vidad si estamos realizando litotricia ultrasónica o cinética. La litotricia con láser de holmio se puede realizar por vía transuretral con instrumentos finos semirrigidos o flexibles, la derivación o trocar suprapúbico se recomienda en cálculos grandes para facilitar la eliminación de partículas y fragmentos, arrastrados por el flujo del líquido de irrigación y para mejorar visión con flujo permanente sin necesidad de detener la litotricia para lavado y extracción de fragmentos, especialmente cuando utilizamos finos endoscopios en pacientes jóvenes o con patología ureteral. Algunos autores en vejigas de gran volumen extraen los cálculos vesicales por vía percutánea con la ayuda de una bolsa similar a la que se utiliza en cirugía laparoscópica (37).

La litotricia con láser de holmio esta especialmente indicada en el tratamiento de la litiasis vesical infantil, Ramakrishnan y cols., han tratado 23 niños de 2-12 años (media de 7.8 años) con cálculos de $0.9-4 \mathrm{~cm}$. de diámetro (media de $2.7 \mathrm{~cm}$.), aplicando energías de (0.6-1.8 $\mathrm{J} /$ pulso a $5-12$ hertzios con fibra de 550 micras, los procedimientos se realizaron en 19-62 mi (media de $38 \mathrm{mi}$ ), nosotros observamos el mayor efecto fototérmico aplicando el láser de holmio a $2500 \mathrm{~mJ}$ y $3 \mathrm{~Hz}$ y fibra de 600 micras. Los cálculos se pulverizaron en fragmentos de 2-3 $\mathrm{mm}$ y en ningún caso observamos litiasis residual, el catéter vesical se retiró en 6-10 horas, con estancia hospitalaria de 2-3 días, no se observaron complicaciones importantes (38). La cistolitotricia suprapúbica ha sido propuesta como alternativa a la cirugía abierta en los pacientes pediátricos con uretra estrecha o patológica o con gran masa litiásica.

Consideramos que hoy, la litotricia vesical endoscópica con láser de holmio si es alternativa terapéutica. A pesar de que existen múltiples opciones de tratamiento endoscópico, la litotricia transuretral con láser de holmio o con energía cinética ofrecen los mejores resultados con un bajo porcentaje de complicaciones, la vía percutánea suprapúbica es altamente efectiva pero debe reservarse para los casos de mayor complejidad.

La cistolitotomía abierta ha sido el tratamiento histórico de los cálculos vesicales con alto porcentaje de éxito, pero en la actualidad su uso es poco frecuente (16), puede indicarse en casos de grandes masas litiásicas, cálculos muy duros, anomalías anatómicas o pacientes con indicación de cirugía abierta por otras patologías relacionadas. La litotricia extracorpórea con ondas de choque sólo se debe considerar como alternativa terapéutica en cálculos primarios menores de $2-3 \mathrm{~cm}^{2}$ o en pacientes con alto riesgo quirúrgico (39).

El tratamiento definitivo de la litiasis vesical es el que corrige sus causas, por lo que se deben tratar las alteraciones hidrodinámicas, neurológicas, evitar el residuo postmiccional y realizar profilaxis o tratamiento precoz de la infección urinaria y de las alteraciones metabólicas (8), valga como ejemplo el caso de Vaidyanathan y cols. en el que demuestran como el tratamiento intermitente con etiodronato controla la litiasis vesical recidivante $(40)$ en un paciente lesionado medular con hipercalciuria por pérdida de masa ósea secundaria a la inmovilización, nosotros en estos casos obtenemos mejor resultado con bifosfonatos de tercera generación (41). 


\section{BIBLIOGRAFÍA Y LECTURAS RECOMENDADAS (*lectura de interés $y$ ** lectura fundamental)}

1. ARRABAL MARTÍN, M.: "Historical, epidemiologic, and therapeutic profile of urolithiasis". Arch. Esp. Urol., 54: 845, 2001

2. SMITH, L.H.: "Medical evolution of urolithiasis, etiologic. Aspect end diagnostic". Urol. Clinic North Am., 1: $241,1974$.

3. SUTOR, D.J.; WOOLEY, S.E.; ILLING, W.: "A geographical end historical survey of the composition of urinary stone". Br. J. Urol., 46: 275, 1974.

4. SALAZAR, R.; ARRABAL, M.: "Tratamiento de la litiasis del tracto urinario inferior". Arrabal, M.; Lancina, J.A.; García, M.: Criterios clínicos y tratamiento actual de la litiasis urinaria. Ed. ENE. Madrid, 1990.

5. ASCI, R.; AYBEK, Z.; SARIKAYA, S. y cols.: "The management of vesical calculi with combined optical mechanical cystolithotripsy and transurethral prostatectomy: Is it safe and effective?". BJU Int., 84: 32, 1999.

6. CURSIO, R.; CHOQUENET, C.: "Iatrogenic bladder stone formation on absorbable suture 3-years after radical prostatectomy". Minerva Urol. Nefro., 54: 127, 2002.

7. MUSTAFA, M.; WADIE, B.S.: "Bladder erosion of tension-free vaginal tape presented as vesical stone; Management and review of literature". Int. Urol. Nephro., 39: 453, 2007.

8. BERGMAN, B.; NYGAARDH, E.: "Follow-up of patients treated for urinary bladder calculi". Int. Urol. Nephro., 22: $331,1990$.

9. KAMBAL, A.: "The relation of urinary bilharziosis to vesical stone in children". Br. J. Urol., 53: 315, 1980.

10. ARRABAL, M.; REINA, M.C.; LANCINA, J.A. y cols.: "Clasificación clínico-terapéutica de la litiasis urinaria". Arch. Esp. Urol., 45: 661, 1992.

11. CIFUENTES DELATTE, L.: "Cirugía urológica endoscópica". Ed. Paz Montalvo SA. Madrid, 1981.

12. DÍAZ, F.: "Tratado de todas las enfermedades del riñón, vejiga, carnosidades de la verga y orina". Edición Facsimil, 1983.

13. MULVANEY, W.P.: "The clinical use of renacidin in urinary calcification". J. Urol., 84: 206, 1960.

14. ROBLES GARCÍA, J.E.; ROSELL COSTA, D.; LONGO ARESO, J. y cols.: "Extracorporeal shockwave lithotripsy (ESWL) for the treatment of bladder lithiasis. A new therapeutic option". Actas Urol. Esp., 14: 456, 1990.

15. ARRABAL MARTÍN, M.: "Grupo de Litiasis de la Associación Española de Urología. Extracorporeal lithotripsy in Spain in the 20th. Century”. Actas Urol. Esp., 24: 699, 2000.

*16. BHATIA, .V.; BIYANI, C.S.: "Vesical lithiasis: Open surgery versus cystolithotripsy versus extracorporeal shock wave therapy". J. Urol., 151: 660, 1994.

17. KOJIMA, Y.; YOSHIMURA, M.; HAYASHI, Y. y cols.: "Extracorporeal shock wave lithotripsy for vesical lithiasis". Urol. Int., 61: 35, 1998.

18. DELAKAS, D.; DASKALOPOULOS, G.; CRANIDIS, A.: "Experience with the Dornier lithotriptor MPL 9000-X for the treatment of vesical lithiasis". Int. Urol. Nephrol., 30: 703, 1998.

19. MILLÁN RODRÍGUEZ, F.; TORNERO RUIZ, J.; LÓPEZ LLAURADÓ, H. y cols.: "Treatment of bladder lithiasis with shock-wave extracorporeal lithotripsy". Actas Urol. Esp., 25: 504, 2001.

20. DUARTE, R.J.; MITRE, A.I.; DÉNES, F.T. y cols.: "Extracorporeal shock wave lithotripsy in children: Results and short-term complications". J. Pediatr., 78: 367, 2002.

21. SALAMA, M.N.; TSUJI, M.; TAMURA, M. y cols.: "Immediate effects of extracorporeal shock waves on the male genital system of rabbit. Preliminary report". Scand J. Urol. Nephro., 32: 251, 1998.

22. PEREZ CASTRO, E.: "Litotricia vesical electrohidráulica". Arch. Esp. Urol., 23: 207, 1970.

23. ZHAOWU, Z.; XIWEN, W.; FENLING, Z.: "Experience with electrohydraulic shockwave lithotripsy in the treatment of vesical calculi”. Br. J. Urol., 61: 498, 1988.

24. GLENN, J.F.: "Bladder stone from litotripsy to ultrasound". Urol. Clin. North Am., 1: 375, 1974.

*25. ARON, M.; GOEL, R.; GAUTAM, G. y cols.: "Percutaneous versus transurethral cystolithotripsy and TURP for large prostates and large vesical calculi: Refinement of technique and updated data". Int. Urol. Nephrol., 39: 173, 2007.

*26. IGLESIAS PRIETO, J.I.; PÉREZ-CASTRO ELLENDT, E.: "Contact lithotripsy. Advantages and disadvantages". Arch. Esp. Urol., 54: 885, 2001.

27. WADHWA, S.N.; HEMAL, A.K.; SHARMA, R.K.: "Intracorporeal lithotripsy with the Swiss lithoclast". Br. J. Urol., 74: 699, 1994.

28. IGLESIAS, J.I.; MANCEBO, J.M.; MASSARRA, J. y cols.: "The use of the EKL-Compact lithotriptor in the endoscopic treatment of ureteral lithiasis". Arch. Esp. Urol., 50: 135, 1997.

*29. RANÉ, A.; KOMMU, S.S.; KANDASWAMY, S.V. y cols.: "Initial clinical evaluation of a new pneumatic intracorporeal lithotripter". BJU Int., 100: 629, 2007.

30. WATSON, G.M,; WICKHAN, J.E.A.: "Initial experience with a pulsed-dye laser for ureteral calculi". Lancet, 1: 1353,1986

31. TSUBOI, N.; HORIUCHI, K.; OKI, M. y cols.: "Clinical treatment of urinary tract stones with Alexandrite laser". Hinyokika Kiyo, 40: 101, 1994.

32. DRETLER, S.P.: "Laser lithotripsy: A review of 20 years of research and clinical applications". Lasers Surg. Med., 8: $341,1988$.

33. WEBER, H.M.; MILLER, K.; RÜSCHOFF, J. y cols.: "Experimental results and initial clinical experiences with the alexandrite laser lithotripter". Urologe A., 29: 304, 1990.

34. MATTIOLI, S.; CREMONA, M.; BENAIM, G. y cols.: "Lithotripsy with a Q-switched alexandrite laser system. Preliminary in vivo and in vitro results". Eur. Urol., 19: 233, 1991.

**35. YATES, J.; ZABBO, A.; PAREEK, G.: “A Comparison of the FREDDY and Holmium Lasers During Ureteroscopic Lithotripsy". Lasers in Surgery and Medicine, 39: 637, 2007.

**36. SHAH, H.N.; HEGDE, S.S.; SHAH, J. y cols.: "Simultaneous transurethral cystolithotripsy with holmium laser enucleation of the prostate: A prospective feasibility study and review of literature". J. U. Int., 99: 595, 2007.

37. MILLER, D.C.; PARK, J.M.: "Percutaneous cystolithotomy using a laparoscopic entrapment sac". Urology, 62: 333, 2003.

**38. RAMAKRISHNAN, P.A.; MEDHAT, M.; AL-BULUSHI, Y.H. y cols.: "Holmium laser cystolithotripsy in children: Initial experience". Can. J. Urol., 12: 2880, 2005.

*39. PAPATSORIS, A.G.; VARKARAKIS, I.; DELLIS, A. y cols.: "Bladder lithiasis: From open surgery to lithotripsy". Urol. Res., 34: 163, 2006.

40. VAIDYANATHAN, S.; WATSON, I.D.; JONSSON, O. y cols.: "Recurrent vesical calculi, hypercalciuria, and biochemical evidence of increased bone resorption in an adult male with paraplegia due to spinal cord injury: Is there a role for intermittent oral disodium etidronate therapy for prevention of calcium phosphate bladder stones?". Spinal Cord., 43: 269, 2005.

41. ARRABAL MARTÍN, M.; DÍAZ DE LA GUARDIA, F.V.; JIMÉNEZ PACHECO, A. y cols.: "The treatment of renal lithiasis with biphosphonates". Arch. Esp. Urol., 60: 745, 2007. 